\title{
Tripled and coincidence fixed point theorems for contractive mappings satisfying $\Phi$-maps in partially ordered metric spaces
}

\author{
Wasfi Shatanawi, Mihai Postolache and Zead Mustafa
}

\begin{abstract}
In this paper, we study some tripled fixed and coincidence point theorems for two mappings $F: X \times X \times X \rightarrow X$ and $g: X \rightarrow X$ satisfying a nonlinear contraction based on $\phi$-maps. Our results extend and improve many existing results in the literature. Also, we introduce an example to support the validity of our results.
\end{abstract}

\section{Introduction and Preliminaries}

The notion of a coupled fixed point for a mapping $F: X \times X \rightarrow X$ was initiated by Gnana Bhaskar and Lakshmikantham [1] and they proved some interesting coupled fixed point theorems, while Ćirić and Lakshmikantham [2] introduced the concept of the coupled fixed point for two mappings $F: X \times X \rightarrow X$ and $g: X \rightarrow X$ and established many existing theorems. Vasile Berinde and Marin Borcut $[3,4]$ initiated the concept of a tripled fixed point of the mapping $F: X \times X \times X \rightarrow X$ and the concept of a tripled coincidence point of the two mappings $F: X \times X \times X \rightarrow X$ and $g: X \rightarrow X$ and extended the results of Gnana Bhaskar and Lakshmikantham [1] and Ćirić and Lakshmikantham [2] to the interesting tripled fixed and coincidence point theorems. For some

Key Words: Tripled fixed point, ordered sets, generalized metric spaces, mixed monotone property.

2010 Mathematics Subject Classification: Primary 54H25, 47H10; Secondary 54E50.

Received: June, 2013.

Revised: October, 2013

Accepted: November, 2013. 
coupled fixed point results in different metric spaces we refer the reader to [5]-[25].

Consistent with Berinde and Borcut [3, 4], we give the following definitions and preliminaries.

Definition 1.1 ([3]). Let $X$ be a nonempty set and $F: X \times X \times X \rightarrow X$ be a given mapping. An element $(x, y, z) \in X \times X \times X$ is called a tripled fixed point of $F$ if

$$
F(x, y, z)=x, F(y, x, y)=y \quad \text { and } \quad F(z, y, x)=z .
$$

Let $(X, d)$ be a metric space. The mapping

$$
\bar{d}: X \times X \times X \rightarrow \mathbb{R}, \quad \bar{d}((x, y, z),(u, v, w))=d(x, u)+d(y, v)+d(z, w),
$$

defines a metric on $X \times X \times X$, which will be denoted for convenience by $d$.

Definition $1.2([3])$. Let $(X, \leq)$ be a partially ordered set and $F: X \times$ $X \times X \rightarrow X$ be a mapping. We say that $F$ has the mixed monotone property if $F(x, y, z)$ is monotone non-decreasing in $x$ and $z$ and is monotone nonincreasing in $y$; that is, for any $x, y, z \in X$,

$$
\begin{array}{rccc}
x_{1}, x_{2} \in X, \quad x_{1} \leq x_{2} \quad \text { implies } & F\left(x_{1}, y, z\right) \leq F\left(x_{2}, y, z\right), \\
y_{1}, y_{2} \in X, \quad y_{1} \leq y_{2} \quad \text { implies } & F\left(x, y_{1}, z\right) \geq F\left(x, y_{2}, z\right),
\end{array}
$$

and

$$
z_{1}, z_{2} \in X, \quad z_{1} \leq z_{2} \quad \text { implies } \quad F\left(x, y, z_{1}\right) \leq F\left(x, y, z_{2}\right) .
$$

The main results of [3] are:

Theorem 1.1 ([3]). Let $(X, \leq)$ be a partially ordered set and $(X, d)$ be a complete metric space. Let $F: X \times X \times X \rightarrow X$ be a continuous mapping such that $F$ has the mixed monotone property. Assume that there exist $j, k, l \in[0,1)$ with $j+k+l<1$ such that

$$
d(F(x, y, z), F(u, v, w)) \leq j d(x, u)+k d(y, v)+l d(z, w)
$$

for all $x, y, z, u, v, w \in X$ with $x \geq u, y \leq v$, and $z \geq w$. If there exist $x_{0}, y_{0}, z_{0} \in X$ such that $x_{0} \leq F\left(x_{0}, y_{0}, z_{0}\right), y_{0} \geq F\left(y_{0}, x_{0}, y_{0}\right)$, and $z_{0} \leq$ $F\left(z_{0}, y_{0}, x_{0}\right)$, then $F$ has a tripled fixed point.

Theorem $1.2([3])$. Let $(X, \leq)$ be a partially ordered set and $(X, d)$ be a complete metric space. Let $F: X \times X \times X \rightarrow X$ be a mapping having the mixed monotone property. Assume that there exist $j, k, l \in[0,1)$ with $j+k+l<1$ such that

$$
d(F(x, y, z), F(u, v, w)) \leq j d(x, u)+k d(y, v)+l d(z, w)
$$


for all $x, y, z, u, v, w \in X$ with $x \geq u, y \leq v$, and $z \geq w$. Assume that $X$ has the following properties:

$i$. if a non-decreasing sequence $x_{n} \rightarrow x$, then $x_{n} \leq x$ for all $n \in \mathbb{N}$,

ii. if a non-increasing sequence $y_{n} \rightarrow y$, then $y_{n} \geq y$ for all $n \in \mathbb{N}$.

If there exist $x_{0}, y_{0}, z_{0} \in X$ such that $x_{0} \leq F\left(x_{0}, y_{0}, z_{0}\right), y_{0} \geq F\left(y_{0}, x_{0}, y_{0}\right)$, and $z_{0} \leq F\left(z_{0}, y_{0}, x_{0}\right)$, then $F$ has a tripled fixed point.

Also, Borcut and Berinde [4] introduced the concept of a tripled coincidence point of mappings $F: X \times X \times X \rightarrow X$ and $g: X \rightarrow X$.

Definition 1.3 ([4]). Let $X$ be a nonempty set. Let $F: X \times X \times X \rightarrow X$ and $g: X \rightarrow X$ be two mappings. An element $(x, y, z) \in X \times X \times X$ is called a tripled coincidence point of $F$ and $g$ if

$$
F(x, y, z)=g x, F(y, x, y)=g y \quad \text { and } \quad F(z, y, x)=g z .
$$

Definition 1.4 ([4]). Let $(X, \leq)$ be a partially ordered set and $F: X \times X \times$ $X \rightarrow X$ be a mapping. We say that $F$ has the mixed g-monotone property if $F(x, y, z)$ is monotone non-decreasing in $x$ and $z$ and is monotone nonincreasing in $y$; that is, for any $x, y, z \in X$,

$$
\begin{array}{rccc}
x_{1}, x_{2} \in X, \quad g x_{1} \leq g x_{2} & \text { implies } & F\left(x_{1}, y, z\right) \leq F\left(x_{2}, y, z\right), \\
y_{1}, y_{2} \in X, \quad g y_{1} \leq g y_{2} & \text { implies } & F\left(x, y_{1}, z\right) \geq F\left(x, y_{2}, z\right),
\end{array}
$$

and

$$
z_{1}, z_{2} \in X, \quad g z_{1} \leq g z_{2} \quad \text { implies } \quad F\left(x, y, z_{1}\right) \leq F\left(x, y, z_{2}\right) .
$$

The main results of [4] is:

Theorem $1.3([4])$. Let $(X, \leq)$ be a partially ordered set and $(X, d)$ be a complete metric space. Let $F: X \times X \times X \rightarrow X$ and $g: X \rightarrow X$ such that $F$ has the mixed $g$-monotone property. Assume that there exist $j, k, l \in[0,1)$ with $j+k+l<1$ such that

$$
d(F(x, y, z), F(u, v, w)) \leq j d(g x, g u)+k d(g y, g v)+l d(g z, g w)
$$

for all $x, y, z, u, v, w \in X$ with $g x \geq g u, g y \leq g v$, and $g z \geq g w$. Syppose that $F(X \times X \times X \subseteq g X, g$ is continuous and commute with $F$ and also suppose either

(a) F is continuous or

(b) $X$ has the following property: 
(i) if a non-decreasing sequence $x_{n} \rightarrow x$, then $x_{n} \leq x$ for all $n$,

(ii) if a non-increasing sequence $y_{n} \rightarrow y$, then $y_{n} \geq y$ for all $n$.

If there exist $x_{0}, y_{0}, z_{0} \in X$ such that $g x_{0} \leq F\left(x_{0}, y_{0}, z_{0}\right), g y_{0} \geq F\left(y_{0}, x_{0}, y_{0}\right)$, and $g z_{0} \leq F\left(z_{0}, y_{0}, x_{0}\right)$, then $F$ and $g$ a tripled coincidence point.

By following Matkowski [26], we let $\Phi$ be the set of all non-decreasing functions $\phi:[0,+\infty) \rightarrow[0,+\infty)$ such that $\lim _{n \rightarrow+\infty} \phi^{n}(t)=0$, for all $t>0$, where $\phi^{n}$ denotes the $n$-th iterate of $\phi$. Then its an easy matter to show that:

(1) $\phi(t)<t$ for all $t>0$,

(2) $\phi(0)=0$.

If $\phi \in \Phi$, then we call $\phi$ to be a $\phi$-map.

In this paper, we utilize the notion of $\phi$-map to prove a number of tripled fixed and coincidence point results for two mapping $F: X \times X \times X \rightarrow X$ and $g: X \rightarrow X$ in ordered metric spaces. Our results generalize Theorems 1.1 and 1.3. Also, we support our results by introducing a nontrivial example satisfying our main results and doesn't satisfy the conditions 1 and 2 of Theorems 1.1 and 1.3 respectively.

\section{Main Results}

Our first result is the following.

Theorem 2.1. Let $(X, \leq)$ be a partially ordered set and $(X, d)$ be a metric space. Let $F: X \times X \times X \rightarrow X, g: X \rightarrow X$ be two mappings. Suppose the following

1. $g X$ is a complete subspace of $X$,

2. $F(X \times X \times X) \subseteq g X$,

3. $F$ has the mixed $g$-monotone property,

4. $F$ is continuous and

5. $g$ is continuous and commute with $F$.

Assume that there exists $\phi \in \Phi$ such that

$$
\begin{aligned}
& d(F(x, y, z), F(u, v, w)) \leq \phi(\max \{d(g x, g u), d(g y, g v), d(g z, g w), \\
& d(F(x, y, z), g x), d(F(z, y, x), g z), d(F(u, v, w), g u), d(F(w, v, u), g w)\}),
\end{aligned}
$$

for all $x, y, z, u, v, w \in X$ with $x \geq u, y \leq v$, and $z \geq w$. If there exist $x_{0}, y_{0}, z_{0} \in X$ such that $g x_{0} \leq F\left(x_{0}, y_{0}, z_{0}\right), g y_{0} \geq F\left(y_{0}, x_{0}, y_{0}\right)$, and $g z_{0} \leq$ $F\left(z_{0}, y_{0}, x_{0}\right)$, then $F$ and $g$ have a tripled coincidence point. 
Proof. Suppose there exist $x_{0}, y_{0}, z_{0} \in X$ such that $g x_{0} \leq F\left(x_{0}, y_{0}, z_{0}\right), g y_{0} \geq$ $F\left(y_{0}, x_{0}, y_{0}\right)$, and $g z_{0} \leq F\left(z_{0}, y_{0}, x_{0}\right)$. Define $g x_{1}=F\left(x_{0}, y_{0}, z_{0}\right), g y_{1}=$ $F\left(y_{0}, x_{0}, y_{0}\right)$ and $g z_{1}=F\left(z_{0}, y_{0}, x_{0}\right)$. Then $g x_{0} \leq g x_{1}, g y_{0} \geq g y_{1}$ and $g z_{0} \leq g z_{1}$. Again, define $g x_{2}=F\left(x_{1}, y_{1}, z_{1}\right), g y_{2}=F\left(y_{1}, x_{1}, y_{1}\right)$ and $g z_{2}=$ $F\left(z_{1}, y_{1}, x_{1}\right)$. Since $F$ has the mixed $g$-monotone property, we have $g x_{0} \leq$ $g x_{1} \leq g x_{2}, g y_{2} \leq g y_{1} \leq g y_{0}$ and $g z_{0} \leq g z_{1} \leq g z_{2}$. Continuing this process, we can construct three sequences $\left(g x_{n}\right),\left(g y_{n}\right)$ and $\left(g z_{n}\right)$ in $X$ such that

$$
\begin{aligned}
& g x_{n}=F\left(x_{n-1}, y_{n-1}, z_{n-1}\right) \leq g x_{n+1}=F\left(x_{n}, y_{n}, z_{n}\right), \\
& g y_{n+1}=F\left(y_{n}, x_{n}, y_{n}\right) \leq g y_{n}=F\left(y_{n-1}, x_{n-1}, y_{n-1}\right),
\end{aligned}
$$

and

$$
g z_{n}=F\left(z_{n-1}, y_{n-1}, x_{n-1}\right) \leq g z_{n+1}=F\left(z_{n}, y_{n}, x_{n}\right) .
$$

If, for some integer $n$, we have $\left(g x_{n+1}, g y_{n+1}, g z_{n+1}\right)=\left(g x_{n}, g y_{n}, g z_{n}\right)$, then $F\left(x_{n}, y_{n}, z_{n}\right)=g x_{n}, F\left(y_{n}, x_{n}, y_{n}\right)=g y_{n}$, and $F\left(z_{n}, y_{n}, x_{n}\right)=g z_{n}$; that is, $\left(x_{n}, y_{n}, z_{n}\right)$ is a tripled coincidence point of $F$. Thus we shall assume that $\left(g x_{n+1}, g y_{n+1}, g z_{n+1}\right) \neq\left(g x_{n}, g y_{n}, g z_{n}\right)$ for all $n \in \mathbb{N}$; that is, we assume that either $g x_{n+1} \neq g x_{n}$ or $g y_{n+1} \neq g y_{n}$ or $g z_{n+1} \neq g z_{n}$. For any $n \in \mathbb{N}$, we have

$$
\begin{aligned}
& d\left(g x_{n+1}, g x_{n}\right) \\
= & d\left(F\left(x_{n}, y_{n}, z_{n}\right), F\left(x_{n-1}, y_{n-1}, z_{n-1}\right)\right. \\
\leq & \phi\left(\operatorname { m a x } \left\{d\left(g x_{n}, g x_{n-1}\right), d\left(g y_{n}, g y_{n-1}\right), d\left(g z_{n}, g z_{n-1}\right),\right.\right. \\
& d\left(F\left(x_{n}, y_{n}, z_{n}\right), g x_{n}\right), d\left(F\left(z_{n}, y_{n}, x_{n}\right), g z_{n}\right), \\
& \left.\left.\left.\left.d\left(F\left(x_{n-1}, y_{n-1}, z_{n-1}\right), g x_{n-1}\right)\right), d\left(F\left(z_{n-1}, y_{n-1}, x_{n-1}\right), g z_{n-1}\right)\right)\right\}\right) \\
= & \phi\left(\operatorname { m a x } \left\{d\left(g x_{n}, g x_{n-1}\right), d\left(g y_{n}, g y_{n-1}\right), d\left(g z_{n}, g z_{n-1}\right),\right.\right. \\
& \left.\left.d\left(g x_{n+1}, g x_{n}\right), d\left(g z_{n+1}, g z_{n}\right)\right\}\right) \\
\leq & \phi\left(\operatorname { m a x } \left\{d\left(g x_{n}, g x_{n-1}\right), d\left(g y_{n}, g y_{n-1}\right), d\left(g z_{n}, g z_{n-1}\right),\right.\right. \\
& \left.\left.d\left(g x_{n+1}, g x_{n}\right), d\left(g y_{n+1}, g y_{n}\right), d\left(g z_{n+1}, g z_{n}\right)\right\}\right),
\end{aligned}
$$




$$
\begin{aligned}
& d\left(g y_{n}, g y_{n+1}\right) \\
= & d\left(F\left(y_{n-1}, x_{n-1}, y_{n-1}\right), F\left(y_{n}, x_{n}, y_{n}\right)\right. \\
\leq & \phi\left(\operatorname { m a x } \left\{d\left(g y_{n-1}, g y_{n}\right), d\left(g x_{n-1}, g x_{n}\right), d\left(F\left(y_{n-1}, x_{n-1}, y_{n-1}\right), g y_{n-1}\right),\right.\right. \\
& \left.\left.d\left(F\left(y_{n}, x_{n}, y_{n}\right), g y_{n}\right)\right\}\right) \\
= & \phi\left(\max \left\{d\left(g y_{n-1}, g y_{n}\right), d\left(g x_{n-1}, g x_{n}\right), d\left(y_{n+1}, y_{n}\right)\right\}\right) \\
\leq & \phi\left(\operatorname { m a x } \left\{d\left(g x_{n}, g x_{n-1}\right), d\left(g y_{n}, g y_{n-1}\right), d\left(g z_{n}, g z_{n-1}\right), d\left(g x_{n+1}, g x_{n}\right),\right.\right. \\
& \left.\left.d\left(g y_{n+1}, g y_{n}\right), d\left(g z_{n+1}, g z_{n}\right)\right\}\right),
\end{aligned}
$$

and

$$
\begin{aligned}
& d\left(g z_{n+1}, g z_{n}\right) \\
= & d\left(F\left(z_{n}, y_{n}, x_{n}\right), F\left(z_{n-1}, y_{n-1}, x_{n-1}\right)\right. \\
\leq & \phi\left(\operatorname { m a x } \left\{d\left(g z_{n}, g z_{n-1}\right), d\left(g y_{n}, g y_{n-1}\right), d\left(g x_{n}, g x_{n-1}\right),\right.\right. \\
& d\left(F\left(z_{n}, y_{n}, x_{n}\right), g z_{n}\right), d\left(F\left(x_{n}, y_{n}, z_{n}\right), g x_{n}\right), \\
& \left.\left.\left.\left.d\left(F\left(z_{n-1}, y_{n-1}, x_{n-1}\right), g z_{n-1}\right)\right), d\left(F\left(x_{n-1}, y_{n-1}, z_{n-1}\right), g x_{n-1}\right)\right)\right\}\right) \\
= & \phi\left(\operatorname { m a x } \left\{d\left(g x_{n}, g x_{n-1}\right), d\left(g y_{n}, g y_{n-1}\right), d\left(g z_{n}, g z_{n-1}\right),\right.\right. \\
& \left.\left.d\left(g x_{n+1}, g x_{n}\right), d\left(g z_{n+1}, g z_{n}\right)\right\}\right) \\
\leq & \phi\left(\operatorname { m a x } \left\{d\left(g x_{n}, g x_{n-1}\right), d\left(g y_{n}, g y_{n-1}\right), d\left(g z_{n}, g z_{n-1}\right),\right.\right. \\
& \left.\left.d\left(g x_{n+1}, g x_{n}\right), d\left(g y_{n+1}, g y_{n}\right), d\left(g z_{n+1}, g z_{n}\right)\right\}\right),
\end{aligned}
$$

From (4), (5), and (6), it follows that

$$
\begin{aligned}
& \max \left\{d\left(g x_{n+1}, g x_{n}\right), d\left(g y_{n}, g y_{n+1}\right), d\left(g z_{n+1}, g z_{n}\right)\right\} \\
\leq & \phi\left(\operatorname { m a x } \left\{d\left(g x_{n}, g x_{n-1}\right), d\left(g y_{n}, g y_{n-1}\right), d\left(g z_{n}, g z_{n-1}\right),\right.\right. \\
& \left.\left.d\left(g x_{n+1}, g x_{n}\right), d\left(g y_{n+1}, g y_{n}\right), d\left(g z_{n+1}, g z_{n}\right)\right\}\right) .
\end{aligned}
$$

If

$$
\begin{gathered}
\max \left\{d\left(g x_{n}, g x_{n-1}\right), d\left(g y_{n}, g y_{n-1}\right), d\left(g z_{n}, g z_{n-1}\right),\right. \\
\left.d\left(g x_{n+1}, g x_{n}\right), d\left(g y_{n+1}, g y_{n}\right), d\left(g z_{n+1}, g z_{n}\right)\right\} \\
=\max \left\{d\left(g x_{n+1}, g x_{n}\right), d\left(g y_{n}, g y_{n+1}\right), d\left(g z_{n+1}, g z_{n}\right)\right\},
\end{gathered}
$$


then from (7) we have

$$
\begin{aligned}
& \max \left\{d\left(g x_{n+1}, g x_{n}\right), d\left(g y_{n}, g y_{n+1}\right), d\left(g z_{n+1}, g z_{n}\right)\right\} \\
\leq & \phi\left(\max \left\{d\left(g x_{n}, g x_{n-1}\right), d\left(g y_{n}, g y_{n-1}\right), d\left(g z_{n}, g z_{n-1}\right)\right\}\right) \\
< & \max \left\{d\left(g x_{n+1}, g x_{n}\right), d\left(g y_{n}, g y_{n+1}\right), d\left(g z_{n+1}, g z_{n}\right)\right\},
\end{aligned}
$$

a contradiction. Thus (7) becomes

$$
\begin{aligned}
& \max \left\{d\left(g x_{n+1}, g x_{n}\right), d\left(g y_{n}, g y_{n+1}\right), d\left(g z_{n+1}, g z_{n}\right)\right\} \\
\leq & \phi\left(\max \left\{d\left(g x_{n}, g x_{n-1}\right), d\left(g y_{n}, g y_{n-1}\right), d\left(g z_{n}, g z_{n-1}\right)\right\}\right) .
\end{aligned}
$$

By repeating (8) $n$-times, we get that

$$
\begin{aligned}
& \max \left\{d\left(g x_{n+1}, g x_{n}\right), d\left(g y_{n}, g y_{n+1}\right), d\left(g z_{n+1}, g z_{n}\right)\right\} \\
\leq & \phi\left(\max \left\{d\left(g x_{n}, g x_{n-1}\right), d\left(g y_{n}, g y_{n-1}\right), d\left(g z_{n}, g z_{n-1}\right)\right\}\right) \\
\leq & \phi^{2}\left(\max \left\{d\left(g x_{n-1}, g x_{n-2}\right), d\left(g y_{n-1}, g y_{n-2}\right), d\left(g z_{n-1}, g z_{n-2}\right)\right\}\right) \\
\vdots & \\
\leq & \phi^{n}\left(\max \left\{d\left(g x_{1}, g x_{0}\right), d\left(g y_{1}, g y_{0}\right), d\left(g z_{1}, g z_{0}\right)\right\}\right) .
\end{aligned}
$$

Now, we shall show that $\left(g x_{n}\right),\left(g y_{n}\right)$, and $\left(g z_{n}\right)$ are Cauchy sequence in $X$. Let $\epsilon>0$. Since

$$
\lim _{n \rightarrow+\infty} \phi^{n}\left(\max \left\{d\left(g x_{1}, g x_{0}\right), d\left(g y_{1}, g y_{0}\right), d\left(g z_{1}, g z_{0}\right)\right\}\right)=0
$$

and $\epsilon>\phi(\epsilon)$, there exist $n_{0} \in \mathbb{N}$ such that

$$
\phi^{n}\left(\max \left\{d\left(g x_{1}, g x_{0}\right), d\left(g y_{1}, g y_{0}\right), d\left(g z_{1}, g z_{0}\right)\right\}\right)<\epsilon-\phi(\epsilon),
$$

for all $n \geq n_{0}$.

This implies that,

$$
\max \left\{d\left(g x_{n+1}, g x_{n}\right), d\left(g y_{n}, g y_{n+1}\right), d\left(g z_{n+1}, g z_{n}\right)\right\}<\epsilon-\phi(\epsilon),
$$

for all $n \geq n_{0}$.

For $m, n \in \mathbb{N}$, we will prove by induction on $m$ that

$$
\max \left\{d\left(g x_{n}, g x_{m}\right), d\left(g y_{n}, g y_{m}\right), d\left(g z_{n}, g z_{m}\right)\right\}<\epsilon,
$$

for all $m \geq n \geq n_{0}$.

For $m=n+1$, (11) is true by using (10) and noting that $\epsilon-\phi(\epsilon)<\epsilon$. Now suppose that (11) holds for $m=k$; that is,

$$
\max \left\{d\left(g x_{n}, g x_{k}\right), d\left(g y_{n}, g y_{k}\right), d\left(g z_{n}, g z_{k}\right)\right\}<\epsilon .
$$


For $m=k+1$, we have

$$
\begin{aligned}
& d\left(g x_{n}, g x_{k+1}\right) \\
\leq & d\left(g x_{n}, g x_{n+1}\right)+d\left(g x_{n+1}, g x_{k+1}\right) \\
\leq & \epsilon-\phi(\epsilon)+d\left(F\left(x_{k}, y_{k}, z_{k}\right), F\left(x_{n}, y_{n}, z_{n}\right)\right) \\
\leq & \epsilon-\phi(\epsilon)+\phi\left(\operatorname { m a x } \left\{d\left(g x_{k}, g x_{n}\right), d\left(g y_{k}, g y_{n}\right), d\left(g z_{k}, g z_{n}\right),\right.\right. \\
& d\left(F\left(x_{k}, y_{k}, z_{k}\right), g x_{k}\right), d\left(F\left(z_{k}, y_{k}, x_{k}\right), g z_{k}\right), \\
& \left.\left.d\left(F\left(x_{n}, y_{n}, z_{n}\right), g x_{n}\right), d\left(F\left(z_{n}, y_{n}, x_{n}\right), g z_{n}\right)\right\}\right) \\
= & \epsilon-\phi(\epsilon)+\phi\left(\operatorname { m a x } \left\{d\left(g x_{n}, g x_{k}\right), d\left(g y_{n}, g y_{k}\right), d\left(g z_{n}, g z_{k}\right), d\left(g x_{n+1}, g x_{n}\right),\right.\right. \\
& \left.\left.d\left(g z_{n+1}, g z_{n}\right), d\left(g x_{k+1}, g x_{k}\right), d\left(g z_{k+1}, g z_{k}\right)\right\}\right) \\
\leq & \epsilon-\phi(\epsilon)+\phi\left(\operatorname { m a x } \left\{d\left(g x_{n}, g x_{k}\right), d\left(g y_{n}, g y_{k}\right), d\left(g z_{n}, g z_{k}\right), d\left(g x_{n+1}, g x_{n}\right),\right.\right. \\
& d\left(g y_{n+1}, g y_{n}\right), d\left(g z_{n+1}, g z_{n}\right), d\left(g x_{k+1}, g x_{k}\right), \\
& \left.\left.d\left(g y_{k+1}, g y_{k}\right), d\left(g z_{k+1}, g z_{k}\right)\right\}\right) .
\end{aligned}
$$

Using (10) and (12), we have

$$
\begin{aligned}
& \max \left\{d\left(g x_{n}, g x_{k}\right), d\left(g y_{n}, g y_{k}\right), d\left(g z_{n}, g z_{k}\right), d\left(g x_{n+1}, g x_{n}\right), d\left(g y_{n+1}, g y_{n}\right),\right. \\
& \left.d\left(g z_{n+1}, g z_{n}\right), d\left(g x_{k+1}, g x_{k}\right), d\left(g y_{k+1}, g y_{k}\right), d\left(g z_{k+1}, g z_{k}\right)\right\} \\
& \leq \max \{\epsilon, \epsilon-\phi(\epsilon)\}=\epsilon .
\end{aligned}
$$

From (13), (14) and the properties of $\phi$, we obtain

$$
d\left(g x_{n}, g x_{n+1}\right)<\epsilon-\phi(\epsilon)+\phi(\epsilon)=\epsilon .
$$

Similarly, we show that

$$
d\left(g y_{n}, g y_{k+1}\right)<\epsilon,
$$

and

$$
d\left(g z_{n}, g z_{k+1}\right)<\epsilon
$$

Hence, we have

$$
\max \left\{d\left(g x_{n}, g x_{k+1}\right), d\left(g y_{n}, g y_{k+1}\right), d\left(g z_{n}, g z_{k+1}\right)\right\}<\epsilon .
$$

Thus (11) holds for all $m \geq n \geq n_{0}$. Hence $\left(g x_{n}\right),\left(g y_{n}\right)$ and $\left(g z_{n}\right)$ are Cauchy sequences in $g X$. 
Since $g X$ is complete, there exist $p, q, r \in g X$ such that $\left(g x_{n}\right),\left(g y_{n}\right)$ and $\left(g z_{n}\right)$ converge to $p, q$, and $r$ respectively. Choose $x, y, z \in X$ such that $p=g x, q=g y$ and $r=g z$. Now, we show that $(p, q, r)$ is a coincidence point of $F$. Since $F, g$ are commute and $F$ is continuous, we have

$$
g g x_{n+1}=g\left(F\left(x_{n}, y_{n}, z_{n}\right)\right)=F\left(g x_{n}, g y_{n}, g z_{n}\right) \rightarrow F(p, q, r) .
$$

Also, since $g$ is continuous and $g x_{n} \rightarrow p$, we have $g g x_{n} \rightarrow g p$. By uniqueness of limit, we get $F(p, q, r)=g p$. Similarly, we show that $g q=F(q, p, q)$ and $g r=F(r, q, p)$. So $(p, q, r)$ is a tripled coincidence point of $F$ and $g$.

By taking $\phi(t)=k t, k \in[0,1)$ in Theorem 2.1, we have the following:

Corollary 2.1. Let $(X, \leq)$ be a partially ordered set and $(X, d)$ be a metric space. Let $F: X \times X \times X \rightarrow X, g: X \rightarrow X$ be two mappings. Suppose the following:

1. $g X$ is a complete subspace of $X$,

2. $F(X \times X \times X) \subseteq g X$,

3. $F$ has the mixed $g$-monotone property,

4. $F$ is continuous and

5. $g$ is continuous and commute with $F$.

Assume that there exists $k \in[0,1)$ such that

$$
\begin{gathered}
d(F(x, y, z), F(u, v, w)) \leq k \max \{d(g x, g u), d(g y, g v), d(g z, g w), \\
d(F(x, y, z), g x), d(F(z, y, x), g z), d(F(u, v, w), g u), d(F(w, v, u), g w)\},
\end{gathered}
$$

for all $x, y, z, u, v, w \in X$ with $x \geq u, y \leq v$, and $z \geq w$. If there exist $x_{0}, y_{0}, z_{0} \in X$ such that $g x_{0} \leq F\left(x_{0}, y_{0}, z_{0}\right), g y_{0} \geq F\left(y_{0}, x_{0}, y_{0}\right)$, and $g z_{0} \leq$ $F\left(z_{0}, y_{0}, x_{0}\right)$, then $F$ and $g$ have a tripled coincidence point.

As a consequence of Corollary 2.1, we have the following:

Corollary 2.2. Let $(X, \leq)$ be a partially ordered set and $(X, d)$ be a metric space. Let $F: X \times X \times X \rightarrow X, g: X \rightarrow X$ be two mappings. Suppose the following

1. $g X$ is a complete subspace of $X$,

2. $F(X \times X \times X) \subseteq g X$,

3. $F$ has the mixed $g$-monotone property,

4. $F$ is continuous and

5. $g$ is continuous and commute with $F$.

Assume that there exist $a_{1}, a_{2}, a_{3}, a_{4}, a_{5}, a_{6}, a_{7} \in[0,1)$ with $\sum_{i=1}^{7} a_{i}<1$ such that

$$
\begin{gathered}
d(F(x, y, z), \\
F(u, v, w)) \leq a_{1} d(g x, g u)+a_{2} d(g y, g v)+a_{3} d(g z, g w) \\
+a_{4} d(F(x, y, z), g x)+a_{5} d(F(z, y, x), g z) \\
+a_{6} d(F(u, v, w), g u)+a_{7} d(F(w, v, u), g w)
\end{gathered}
$$


for all $x, y, z, u, v, w \in X$ with $x \geq u, y \leq v$, and $z \geq w$. If there exist $x_{0}, y_{0}, z_{0} \in X$ such that $g x_{0} \leq F\left(x_{0}, y_{0}, z_{0}\right), g y_{0} \geq F\left(y_{0}, x_{0}, y_{0}\right)$, and $g z_{0} \leq$ $F\left(z_{0}, y_{0}, x_{0}\right)$, then $F$ and $g$ have a tripled coincidence point.

By taking $g=i_{X}$ (the identity mapping on $X$ ) in Theorem 2.1, Corollary 2.1 and Corollary 2.2, we have the following:

Corollary 2.3. Let $(X, \leq)$ be a partially ordered set and $(X, d)$ be a complete metric space. Let $F: X \times X \times X \rightarrow X$ be a continuous mapping having the mixed monotone property. Assume that there exists $\phi \in \Phi$ such that

$$
\begin{gathered}
d(F(x, y, z), F(u, v, w)) \leq \phi(\max \{d(x, u), d(y, v), d(z, w), d(F(x, y, z), x), \\
d(F(z, y, x), z), d(F(u, v, w), u), d(F(w, v, u), w)\})
\end{gathered}
$$

for all $x, y, z, u, v, w \in X$ with $x \geq u, y \leq v$, and $z \leq w$. If there exist $x_{0}, y_{0}, z_{0} \in X$ such that $x_{0} \leq F\left(x_{0}, y_{0}, z_{0}\right), y_{0} \geq F\left(y_{0}, x_{0}, y_{0}\right)$, and $z_{0} \leq$ $F\left(z_{0}, y_{0}, x_{0}\right)$, then $F$ has a tripled fixed point.

Corollary 2.4. Let $(X, \leq)$ be a partially ordered set and $(X, d)$ be a complete metric space. Let $F: X \times X \times X \rightarrow X$ be a continuous mapping having the mixed monotone property. Assume that there exists $k \in[0,1)$ such that

$$
\begin{gathered}
d(F(x, y, z), F(u, v, w)) \leq k \max \{d(x, u), d(y, v), d(z, w), d(F(x, y, z), x), \\
d(F(z, y, x), z), d(F(u, v, w), u), d(F(w, v, u), w)\}
\end{gathered}
$$

for all $x, y, z, u, v, w \in X$ with $x \geq u, y \leq v$, and $z \leq w$. If there exist $x_{0}, y_{0}, z_{0} \in X$ such that $x_{0} \leq F\left(x_{0}, y_{0}, z_{0}\right), y_{0} \geq F\left(y_{0}, x_{0}, y_{0}\right)$, and $z_{0} \leq$ $F\left(z_{0}, y_{0}, x_{0}\right)$, then $F$ has a tripled fixed point.

Corollary 2.5. Let $(X, \leq)$ be a partiallyy ordered set and $(X, d)$ be a complete metric space. Let $F: X \times X \times X \rightarrow X$ be a continuous mapping having the mixed monotone property. Assume that there exists $a_{1}, a_{2}, a_{3}, a_{4}, a_{5}, a_{6}, a_{7}$ in $[0,1)$ with $\sum_{i=1}^{7} a_{i}<1$ such that

$$
\begin{gathered}
d(F(x, y, z), F(u, v, w)) \leq a_{1} d(x, u)+a_{2} d(y, v)+a_{3} d(z, w)+ \\
a_{4} d(F(x, y, z), x)+a_{5} d(F(z, y, x), z)+a_{6} d(F(u, v, w), u)+a_{7} d(F(w, v, u), w),
\end{gathered}
$$

for all $x, y, z, u, v, w \in X$ with $x \geq u, y \leq v$, and $z \leq w$. If there exist $x_{0}, y_{0}, z_{0} \in X$ such that $x_{0} \leq F\left(x_{0}, y_{0}, z_{0}\right), y_{0} \geq F\left(y_{0}, x_{0}, y_{0}\right)$, and $z_{0} \leq$ $F\left(z_{0}, y_{0}, x_{0}\right)$, then $F$ has a tripled fixed point.

By adding an additional hypotheses, the continuity of $F$ and $g$ in Theorem 2.1 can be dropped. 
Theorem 2.2. Let $(X, \leq)$ be a partially ordered set and $(X, d)$ be a complete metric space. Let $F: X \times X \times X \rightarrow X$ and $g: X \rightarrow X$ be two mappings. Suppose that there exists $\phi \in \Phi$ such that

$$
\begin{gathered}
d(F(x, y, z), F(u, v, w)) \leq \phi(\max \{d(g x, g u), d(g y, g v), d(g z, g w), \\
d(F(x, y, z), g x), d(F(z, y, x), g z), d(F(u, v, w), g u), d(F(w, v, u), g w)\}),
\end{gathered}
$$

for all $x, y, z, u, v, w \in X$ with $g x \geq g u, g y \leq g v$, and $g z \leq g w$. Suppose the following:

1. $g X$ is a complete subspace of $X$,

2. $F(X \times X \times X) \subseteq g X$,

3. $F$ has the mixed $g$-monotone property,

4. $F$ and $g$ are commute.

Also, assume that $X$ has the following properties:

$i$. if a non-decreasing sequence $x_{n} \rightarrow x$, then $x_{n} \leq x$ for all $n \in \mathbb{N}$,

ii. if a non-increasing sequence $y_{n} \rightarrow y$, then $y_{n} \geq y$ for all $n \in \mathbb{N}$.

If there exist $x_{0}, y_{0}, z_{0} \in X$ such that $g x_{0} \leq F\left(x_{0}, y_{0}, z_{0}\right)$, $g y_{0} \geq F\left(y_{0}, x_{0}, y_{0}\right)$, and $g z_{0} \leq F\left(z_{0}, y_{0}, x_{0}\right)$, then $F$ and $g$ have a tripled coincidence point.

Proof. By following the same process in Theorem 2.1, we construct three Cauchy sequences $\left(g x_{n}\right),\left(g y_{n}\right)$ and $\left(g z_{n}\right)$ in $g X$ with

$$
\begin{aligned}
& g x_{1} \leq g x_{2} \leq \ldots \leq g x_{n} \leq \ldots, \\
& g y_{1} \geq g y_{2} \geq \ldots \geq g y_{n} \geq \ldots,
\end{aligned}
$$

and

$$
g z_{1} \leq g z_{2} \leq \ldots \leq g z_{n} \leq \ldots
$$

such that $g x_{n} \rightarrow p=g x \in g X, g y_{n} \rightarrow q=g y \in g X$, and $g z_{n} \rightarrow r=g z \in g X$, where $x, y, z \in X$. By the hypotheses on $X$, we have $g x_{n} \leq g x, g y_{n} \geq g y$ and $g z_{n} \leq g z$ for all $n \in \mathbb{N}$. From (15), we have

$$
\begin{aligned}
& d\left(F(x, y, z), x_{n+1}\right) \\
= & d\left(F(x, y, z), F\left(x_{n}, y_{n}, z_{n}\right)\right) \\
\leq & \phi\left(\operatorname { m a x } \left\{d\left(x, x_{n}\right), d\left(y, y_{n}\right), d\left(z, z_{n}\right), d(F(x, y, z), g x), d(F(z, y, x), g z),\right.\right. \\
& \left.\left.d\left(F\left(x_{n}, y_{n}, z_{n}\right), g x_{n}\right), d\left(F\left(z_{n}, y_{n}, x_{n}\right), g z_{n}\right)\right\}\right),
\end{aligned}
$$




$$
\begin{aligned}
= & \phi\left(\operatorname { m a x } \left\{d\left(g x, g x_{n}\right), d\left(g y, g y_{n}\right), d\left(g z, g z_{n}\right), d(F(x, y, z), g x),\right.\right. \\
& \left.\left.d(F(z, y, x), g z), d\left(g x_{n+1}, g x_{n}\right), d\left(g z_{n+1}, g z_{n}\right)\right\}\right), \\
\leq & \phi\left(\operatorname { m a x } \left\{d\left(g x, g x_{n}\right), d\left(g y, g y_{n}\right), d\left(g z, g z_{n}\right), d(F(x, y, z), g x),\right.\right. \\
& d(F(z, y, x), g z), d(F(y, x, y), g y), d\left(g x_{n+1}, g x_{n}\right), \\
& \left.\left.d\left(g y_{n+1}, g y_{n}\right), d\left(g z_{n+1}, g z_{n}\right)\right\}\right),
\end{aligned}
$$

$$
\begin{aligned}
& d\left(y_{n+1}, F(y, x, y)\right) \\
= & d\left(F\left(y_{n}, x_{n}, y_{n}\right), F(y, x, y)\right) \\
\leq & \phi\left(\max \left\{d\left(y_{n}, y\right), d\left(x_{n}, x\right), d\left(F\left(y_{n}, x_{n}, y_{n}\right), g y_{n}\right), d(F(y, x, y), g y)\right\}\right) \\
= & \phi\left(\max \left\{d\left(g y_{n}, g y\right), d\left(g x_{n}, g x\right), d\left(g y_{n+1}, g y_{n}\right), d(F(y, x, y), g y)\right\}\right) \\
\leq & \phi\left(\operatorname { m a x } \left\{d\left(g x, g x_{n}\right), d\left(g y, g y_{n}\right), d\left(g z, g z_{n}\right),\right.\right. \\
& d(F(x, y, z), g x), d(F(z, y, x), g z), d(F(y, x, y), g y) \\
& \left.\left.d\left(g x_{n+1}, g x_{n}\right), d\left(g y_{n+1}, g y_{n}\right), d\left(g z_{n+1}, g z_{n}\right)\right\}\right)
\end{aligned}
$$

and

$$
\begin{aligned}
& d\left(F(z, y, x), z_{n+1}\right) \\
= & d\left(F(z, y, x), F\left(z_{n}, y_{n}, x_{n}\right)\right) \\
\leq & \phi\left(\operatorname { m a x } \left\{d\left(z, z_{n}\right), d\left(y, y_{n}\right), d\left(x, x_{n}\right), d(F(z, y, x), g z), d(F(x, y, z), g x),\right.\right. \\
& \left.\left.d\left(F\left(z_{n}, y_{n}, x_{n}\right), g z_{n}\right), d\left(F\left(x_{n}, y_{n}, z_{n}\right), g x_{n}\right)\right\}\right), \\
\leq & \phi\left(\operatorname { m a x } \left\{d\left(g z, g z_{n}\right), d\left(g y, g y_{n}\right), d\left(g x, g x_{n}\right), d(F(z, y, x), g z),\right.\right. \\
& \left.\left.d(F(x, y, z), g x), d\left(g z_{n+1}, g z_{n}\right), d\left(g x_{n+1}, g x_{n}\right)\right\}\right), \\
\leq \quad & \phi\left(\operatorname { m a x } \left\{d\left(g x, g x_{n}\right), d\left(g y, g y_{n}\right), d\left(g z, g z_{n}\right), d(F(x, y, z), g x),\right.\right. \\
& d(F(z, y, x), g z), d(F(y, x, y), g y), d\left(g x_{n+1}, g x_{n}\right), \\
& \left.\left.d\left(g y_{n+1}, g y_{n}\right), d\left(g z_{n+1}, g z_{n}\right)\right\}\right) .
\end{aligned}
$$


From (16)-(18), we have

$$
\begin{aligned}
& \max \left\{d\left(F(x, y, z), x_{n+1}\right), d\left(y_{n+1}, F(y, x, y)\right), d\left(F(z, y, x), z_{n+1}\right)\right\} \\
\leq & \phi\left(\operatorname { m a x } \left\{d\left(g x, g x_{n}\right), d\left(g y, g y_{n}\right), d\left(g z, g z_{n}\right), d(F(x, y, z), g x),\right.\right. \\
& d(F(z, y, x), g z), d(F(y, x, y), g y), d\left(g x_{n+1}, g x_{n}\right), \\
& \left.\left.d\left(g y_{n+1}, g y_{n}\right), d\left(g z_{n+1}, g z_{n}\right)\right\}\right) .
\end{aligned}
$$

Our claim is:

$$
\max \{d(F(x, y, z), g x), d(g y, F(y, x, y)), d(F(z, y, x), g z)\}=0
$$

To prove our claim, suppose that

$$
\max \{d(F(x, y, z), g x), d(g y, F(y, x, y)), d(F(z, y, x), g z)\} \neq 0 .
$$

Let

$$
\epsilon=\max \{d(F(x, y, z), g x), d(g y, F(y, x, y)), d(F(z, y, x), g z)\} .
$$

Since $\epsilon>0, d\left(g x, g x_{n}\right) \rightarrow 0, d\left(g y, g y_{n}\right) \rightarrow 0, d\left(g z, g z_{n}\right) \rightarrow 0, d\left(g x_{n}, g x_{n+1}\right) \rightarrow$ $0, d\left(g y_{n}, g y_{n+1}\right) \rightarrow 0$, and $d\left(g z_{n}, g z_{n+1}\right) \rightarrow 0$, we choose $n_{0} \in \mathbb{N}$ such that

$$
\begin{aligned}
d\left(x, x_{n}\right) & <\frac{\epsilon}{2} \text { for all } n \geq n_{0}, \\
d\left(y, y_{n}\right) & <\frac{\epsilon}{2} \text { for all } n \geq n_{0}, \\
d\left(z, z_{n}\right) & <\frac{\epsilon}{2} \text { for all } n \geq n_{0}, \\
d\left(x_{n}, x_{n+1}\right) & <\frac{\epsilon}{2} \text { for all } n \geq n_{0}, \\
d\left(y_{n}, y_{n+1}\right) & <e \frac{\epsilon}{2} \text { for all } n \geq n_{0},
\end{aligned}
$$

and

$$
d\left(z_{n}, z_{n+1}\right)<\frac{\epsilon}{2} \text { for all } n \geq n_{0}
$$

Thus (19) becomes

$$
\begin{aligned}
& \max \left\{d\left(F(x, y, z), x_{n+1}\right), d\left(y_{n+1}, F(y, x, y)\right), d\left(F(z, y, x), z_{n+1}\right)\right\} \\
\leq & \phi\left(\max \left\{\frac{\epsilon}{2}, d(F(x, y, z), g x), d(F(z, y, x), g z), d(F(y, x, y), g y)\right\}\right) \\
= & \phi(\max \{d(F(x, y, z), g x), d(F(z, y, x), g z), d(F(y, x, y), g y)\})
\end{aligned}
$$

for all $n \geq n_{0}$. 
Letting $n \rightarrow+\infty$ in (20) it follows that

$$
\begin{aligned}
& \max \{d(F(x, y, z), g x), d(g y, F(y, x, y)), d(F(z, y, x), g z)\} \\
\leq & \phi(\max \{d(F(x, y, z), g x), d(F(z, y, x), g z), d(F(y, x, y), g y)\}) \\
< & \max \{d(F(x, y, z), g x), d(g y, F(y, x, y)), d(F(z, y, x), g z)\},
\end{aligned}
$$

a contradiction. Therefore

$$
\max \{d(F(x, y, z), g x), d(g y, F(y, x, y)), d(F(z, y, x), g z)\}=0
$$

and hence $d(F(x, y, z), g x)=0, d(g y, F(y, x, y))=0$ and $d(F(z, y, x), g z)=0$. Thus $F(x, y, z)=g x, F(y, x, y)=g y$ and $F(z, y, x)=g z$; that is $(x, y, z)$ is a tripled fixed point of $F$ and $g$.

By taking $\phi(t)=k t, k \in[0,1)$ in Theorem 2.2, we have the following:

Corollary 2.6. Let $(X, \leq)$ be a partially ordered set and $(X, d)$ be a complete metric space. Let $F: X \times X \times X \rightarrow X$ and $g: X \rightarrow X$ be two mappings. Suppose that there exists $k \in[0,1)$ such that

$$
\begin{aligned}
& d(F(x, y, z), F(u, v, w)) \\
\leq \quad & k \max \{d(g x, g u), d(g y, g v), d(g z, g w), d(F(x, y, z), g x), \\
& d(F(z, y, x), g z), d(F(u, v, w), g u), d(F(w, v, u), g w)\},
\end{aligned}
$$

for all $x, y, z, u, v, w \in X$ with $g x \geq g u, g y \leq g v$, and $g z \leq g w$. Suppose the following:

1. $g X$ is a complete subspace of $X$,

2. $F(X \times X \times X) \subseteq g X$,

3. $F$ has the mixed $g$-monotone property,

4. $F$ and $g$ are commute.

Also, assume that $X$ has the following properties:

$i$. if a non-decreasing sequence $x_{n} \rightarrow x$, then $x_{n} \leq x$ for all $n \in \mathbb{N}$,

ii. if a non-increasing sequence $y_{n} \rightarrow y$, then $y_{n} \geq y$ for all $n \in \mathbb{N}$.

If there exist $x_{0}, y_{0}, z_{0} \in X$ such that $g x_{0} \leq F\left(x_{0}, y_{0}, z_{0}\right)$, $g y_{0} \geq F\left(y_{0}, x_{0}, y_{0}\right)$, and $g z_{0} \leq F\left(z_{0}, y_{0}, x_{0}\right)$, then $F$ and $g$ have a tripled coincidence point.

As a consequence result of Corollary 2.6, we have

Corollary 2.7. Let $(X, \leq)$ be a partially ordered set and $(X, d)$ be a complete metric space. Let $F: X \times X \times X \rightarrow X$ and $g: X \rightarrow X$ be two mappings. 
Suppose that there exist $a_{1}, a_{2}, a_{3}, a_{4}, a_{5}, a_{6}, a_{7} \in[0,1)$ with $\sum_{i=1}^{7} a_{i}<1$ such that

$$
\begin{aligned}
& d(F(x, y, z), F(u, v, w)) \\
\leq \quad & a_{1} d(g x, g u)+a_{2} d(g y, g v)+a_{3} d(g z, g w)+a_{4} d(F(x, y, z), g x)+ \\
& a_{5} d(F(z, y, x), g z)+d a_{6}(F(u, v, w), g u)+a_{7} d(F(w, v, u), g w),
\end{aligned}
$$

for all $x, y, z, u, v, w \in X$ with $g x \geq g u, g y \leq g v$, and $g z \leq g w$. Suppose the following:

1. $g X$ is a complete subspace of $X$,

2. $F(X \times X \times X) \subseteq g X$,

3. $F$ has the mixed $g$-monotone property,

4. F and $g$ are commute.

Also, assume that $X$ has the following properties:

$i$. if a non-decreasing sequence $x_{n} \rightarrow x$, then $x_{n} \leq x$ for all $n \in \mathbb{N}$,

ii. if a non-increasing sequence $y_{n} \rightarrow y$, then $y_{n} \geq y$ for all $n \in \mathbb{N}$.

If there exist $x_{0}, y_{0}, z_{0} \in X$ such that $g x_{0} \leq F\left(x_{0}, y_{0}, z_{0}\right)$, $g y_{0} \geq F\left(y_{0}, x_{0}, y_{0}\right)$, and $g z_{0} \leq F\left(z_{0}, y_{0}, x_{0}\right)$, then $F$ and $g$ have a tripled coincidence point.

By taking $g=i_{X}$ (the identity mapping on $X$ ) in Theorem 2.2, Corollary 2.6 and Corollary 2.7, we have the following:

Corollary 2.8. Let $(X, \leq)$ be a partially ordered set and $(X, d)$ be a complete metric space. Let $F: X \times X \times X \rightarrow X$ be a mapping having the mixed monotone property. Assume that there exists $\phi \in \Phi$ such that

$$
\begin{aligned}
d(F(x, y, z), F(u, v, w)) & \leq \phi(\max \{d(x, u), d(y, v), d(z, w), d(F(x, y, z), x), \\
& d(F(z, y, x), z), d(F(u, v, w), u), d(F(w, v, u), w)\})
\end{aligned}
$$

for all $x, y, z, u, v, w \in X$ with $x \geq u, y \leq v$, and $z \leq w$. Assume also that $X$ has the following properties:

$i$. if a non-decreasing sequence $x_{n} \rightarrow x$, then $x_{n} \leq x$ for all $n \in \mathbb{N}$,

ii. if a non-increasing sequence $y_{n} \rightarrow y$, then $y_{n} \geq y$ for all $n \in \mathbb{N}$.

If there exist $x_{0}, y_{0}, z_{0} \in X$ such that $x_{0} \leq F\left(x_{0}, y_{0}, z_{0}\right), y_{0} \geq F\left(y_{0}, x_{0}, y_{0}\right)$, and $z_{0} \leq F\left(z_{0}, y_{0}, x_{0}\right)$, then $F$ has a tripled fixed point.

Corollary 2.9. Let $(X, \leq)$ be a partially ordered set and $(X, d)$ be a complete metric space. Let $F: X \times X \times X \rightarrow X$ be a mapping having the mixed monotone property. Assume that there exists $k \in[0,1)$ such that

$$
\begin{array}{r}
d(F(x, y, z), F(u, v, w)) \leq k \max \{d(x, u), d(y, v), d(z, w), d(F(x, y, z), x), \\
d(F(z, y, x), z), d(F(u, v, w), u), d(F(w, v, u), w)\}
\end{array}
$$


for all $x, y, z, u, v, w \in X$ with $x \geq u, y \leq v$, and $z \leq w$. Assume also that $X$ has the following properties:

$i$. if a non-decreasing sequence $x_{n} \rightarrow x$, then $x_{n} \leq x$ for all $n \in \mathbb{N}$,

ii. if a non-increasing sequence $y_{n} \rightarrow y$, then $y_{n} \geq y$ for all $n \in \mathbb{N}$.

If there exist $x_{0}, y_{0}, z_{0} \in X$ such that $x_{0} \leq F\left(x_{0}, y_{0}, z_{0}\right), y_{0} \geq F\left(y_{0}, x_{0}, y_{0}\right)$, and $z_{0} \leq F\left(z_{0}, y_{0}, x_{0}\right)$, then $F$ has a tripled fixed point.

Corollary $2.10([3])$. Let $(X, \leq)$ be a partially ordered set and $(X, d)$ be a complete metric space. Let $F: X \times X \times X \rightarrow X$ be a mapping having the mixed monotone property. Assume that there exist $a_{1}, a_{2}, a_{3}, a_{4}, a_{5}, a_{6}, a_{7} \in[0,1)$ with $\sum_{i=1}^{7} a_{i}<1$ such that

$$
d(F(x, y, z), F(u, v, w)) \leq a_{1} d(x, u)+a_{2} d(y, v)+a_{3} d(z, w)+
$$

$a_{4} d(F(x, y, z), x)+a_{5} d(F(z, y, x), z)+a_{6} d(F(u, v, w), u)+a_{7} d(F(w, v, u), w)$,

for all $x, y, z, u, v, w \in X$ with $x \geq u, y \leq v$, and $z \geq w$. Assume that $X$ has the following properties:

$i$. if a non-decreasing sequence $x_{n} \rightarrow x$, then $x_{n} \leq x$ for all $n \in \mathbb{N}$,

ii. if a non-increasing sequence $y_{n} \rightarrow y$, then $y_{n} \geq y$ for all $n \in \mathbb{N}$.

If there exist $x_{0}, y_{0}, z_{0} \in X$ such that $x_{0} \leq F\left(x_{0}, y_{0}, z_{0}\right), y_{0} \geq F\left(y_{0}, x_{0}, y_{0}\right)$, and $z_{0} \leq F\left(z_{0}, y_{0}, x_{0}\right)$, then $F$ has a tripled fixed point.

Now, we prove a uniqueness theorem for a tripled fixed point.

Theorem 2.3. In addition to the hypotheses of Theorem 2.1 (respectfully Theorem 2.2) suppose that

$$
\left[\left(g x_{0} \leq g y_{0}\right) \wedge\left(g z_{0} \leq g y_{0}\right)\right] \vee\left[\left(g y_{0} \leq g x_{0}\right) \wedge\left(g y_{0} \leq g z_{0}\right)\right]
$$

Then $g x=g y=g z$.

Proof. Suppose to the contrary, that is $g x \neq g y$ or $g y \neq g z$ or $g x \neq g z$. Let

$$
\epsilon=\max \{d(g x, g y), d(g x, g z), d(g y, g z)\} .
$$

Since $\epsilon>0, d\left(g x_{n}, g x_{n+1}\right) \rightarrow 0, d\left(g y_{n}, g y_{n+1}\right) \rightarrow 0$ and $d\left(g z_{n}, g z_{n+1}\right) \rightarrow 0$ there exist $n_{0}>0$ such that

$$
\begin{aligned}
& d\left(g x_{n}, g x_{n+1}\right)<\frac{\epsilon}{2} \text { for all } n \geq n_{0} \\
& d\left(g y_{n}, g y_{n+1}\right)<\frac{\epsilon}{2} \text { for all } n \geq n_{0}
\end{aligned}
$$

and

$$
d\left(g z_{n}, g z_{n+1}\right)<\frac{\epsilon}{2} \text { for all } n \geq n_{0}
$$


Without loss of generality, we may assume that $g x_{0} \leq g y_{0}$ and $g z_{0} \leq g y_{0}$. By the mixed monotone property of $F$, we have $g x_{n} \leq g y_{n}$ and $g z_{n} \leq g y_{n}$ for all $n \in \mathbb{N}$. Thus by (3), we have

$$
\begin{aligned}
& d\left(g y_{n+1}, g x_{n+1}\right) \\
= & d\left(F\left(y_{n}, x_{n}, y_{n}\right), F\left(x_{n}, y_{n}, z_{n}\right)\right) \\
\leq & \phi\left(\operatorname { m a x } \left\{d\left(g y_{n}, g x_{n}\right), d\left(g y_{n}, g z_{n}\right), d\left(F\left(y_{n}, x_{n}, y_{n}\right), g y_{n}\right),\right.\right. \\
& \left.\left.d\left(F\left(x_{n}, y_{n}, z_{n}\right), g x_{n}\right), d\left(F\left(z_{n}, y_{n}, x_{n}\right), g z_{n}\right)\right\}\right) \\
\leq & \phi\left(\max \left\{d\left(g y_{n}, g x_{n}\right), d\left(g y_{n}, g z_{n}\right), d\left(g y_{n+1}\right), g y_{n}\right)\right. \\
& \left.\left.d\left(g x_{n+1}, g x_{n}\right), d\left(g z_{n+1}, g z_{n}\right)\right\}\right)
\end{aligned}
$$

and

$$
\begin{aligned}
& d\left(g y_{n+1}, g z_{n+1}\right) \\
= & d\left(F\left(y_{n}, x_{n}, y_{n}\right), F\left(z_{n}, y_{n}, x_{n}\right)\right) \\
\leq & \phi\left(\operatorname { m a x } \left\{d\left(g y_{n}, g x_{n}\right), d\left(g y_{n}, g z_{n}\right), d\left(F\left(y_{n}, x_{n}, y_{n}\right), g y_{n}\right)\right.\right. \\
& \left.\left.d\left(F\left(x_{n}, y_{n}, z_{n}\right), g x_{n}\right), d\left(F\left(z_{n}, y_{n}, x_{n}\right), g z_{n}\right)\right\}\right) \\
\leq & \phi\left(\max \left\{d\left(g y_{n}, g x_{n}\right), d\left(g y_{n}, g z_{n}\right), d\left(g y_{n+1}\right), g y_{n}\right)\right. \\
& \left.\left.d\left(g x_{n+1}, g x_{n}\right), d\left(g z_{n+1}, g z_{n}\right)\right\}\right)
\end{aligned}
$$

By (21) and (22), we have

$$
\begin{aligned}
& \max \left\{d\left(g y_{n+1}, g x_{n+1}\right), d\left(g y_{n+1}, g z_{n+1}\right)\right\} \\
\leq & \phi\left(\max \left\{d\left(g y_{n}, g x_{n}\right), d\left(g y_{n}, g z_{n}\right), d\left(g y_{n+1}\right), g y_{n}\right),\right. \\
& \left.\left.d\left(g x_{n+1}, g x_{n}\right), d\left(g z_{n+1}, g z_{n}\right)\right\}\right) .
\end{aligned}
$$

For $n \geq n_{0}$, we have

$$
\begin{aligned}
& \max \left\{d\left(g y_{n+1}, g x_{n+1}\right), d\left(g y_{n+1}, g z_{n+1}\right)\right\} \\
\leq & \phi\left(\max \left\{d\left(g y_{n}, g x_{n}\right), d\left(g y_{n}, g z_{n}\right), \frac{\epsilon}{2}\right\}\right) \\
\leq & \phi^{2}\left(\max \left\{d\left(g y_{n-1}, g x_{n-1}\right), d\left(g y_{n-1}, g z_{n-1}\right), \frac{\epsilon}{2}\right\}\right)
\end{aligned}
$$




$$
\begin{aligned}
& \leq \phi^{3}\left(\max \left\{d\left(g y_{n-2}, g x_{n-2}\right), d\left(g y_{n-2}, g z_{n-2}\right), \frac{\epsilon}{2}\right\}\right) \\
& \vdots \\
& \leq \phi^{n-n_{0}}\left(\max \left\{d\left(g y_{n_{0}}, g x_{n_{0}}\right), d\left(g y_{n_{0}}, g z_{n_{0}}\right), \frac{\epsilon}{2}\right\}\right) .
\end{aligned}
$$

On letting $n \rightarrow+\infty$ in (23) and using the property of $\phi$ and the fact that $d$ is continuous on its variables, we get that $\max \{d(g y, g x), d(g y, g z)\}=0$. Hence $g y=g z=g x$, a contradiction.

Corollary 2.11. In addition to the hypotheses of Corollary 2.1 (respectfully Corollary 2.6) suppose that

$$
\left[\left(g x_{0} \leq g y_{0}\right) \wedge\left(g z_{0} \leq g y_{0}\right)\right] \vee\left[\left(g y_{0} \leq g x_{0}\right) \wedge\left(g y_{0} \leq g z_{0}\right)\right] .
$$

Then $g x=g y=g z$.

Now, we introduce an example to support the useability of our results:

Example 2.1. Let $X=[0,1]$ with usual ordered. Define $d: X \times X \rightarrow X$ by $d(x, y)=|x-y|$. Define $g: X \rightarrow X$ and $F: X \times X \times X \rightarrow X$ by $g x=\frac{3}{4} x$, and

$$
F(x, y, z)= \begin{cases}0, & y \geq \min \{x, z\} \\ \frac{1}{3}(\min \{x, z\}-y), & y<\min \{x, z\}\end{cases}
$$

Then:

1. $g X$ is a complete subspace of $X$.

2. $F(X \times X \times X) \subseteq g X$.

3. $F$ and $g$ are commute.

4. $F$ has the mixed $g$-monotone property.

5. For $x, y, z, u, v, w \in X$ we have

$$
\begin{aligned}
& d(F(x, y, z), F(u, v, w)) \\
\leq & \frac{8}{9} \max \{d(g x, g u), d(g y, g v), d(g z, g w), d(F(x, y, z), g x), \\
& d(F(z, y, x), g x), d(F(u, v, w), g u), d(F(w, v, u), g w)\}
\end{aligned}
$$

holds, for all $g x \geq g u, g y \leq g v$ and $g z \geq g w$.

6. There are no $j, k, l \in[0,1)$ with $j+k+l<1$ such that

$$
d(F(x, y, z), F(u, v, w)) \leq j d(x, u)+k d(y, v)+l d(z, w)
$$

holds for all $x \geq u, y \leq v$ and $z \geq w$.

7. There are no $j, k, l \in[0,1)$ with $j+k+l<1$ such that

$$
d(F(x, y, z), F(u, v, w)) \leq j d(g x, g u)+k d(g y, g v)+l d(g z, g w)
$$

holds for all $g x \geq g u, g y \leq g v$ and $g z \geq g w$. 
Proof. The proof of (1), (2) and (3) are clear. To prove (4), let $x, y, z \in X$. To show that $F(x, y, z)$ is monotone $g$-non-decreasing in $x$, let $x_{1}, x_{2} \in X$ with $g x_{1} \leq g x_{2}$. Then $\frac{3}{4} x_{1} \leq \frac{3}{4} x_{2}$ and hence $x_{1} \leq x_{2}$.

If $y \geq \min \left\{x_{1}, z\right\}$, then $F\left(x_{1}, y, z\right)=0 \leq F\left(x_{2}, y, z\right)$.

If $y<\min \left\{x_{1}, z\right\}$, then

$$
F\left(x_{1}, y, z\right)=\frac{1}{3}\left(\min \left\{x_{1}, z\right\}-y\right) \leq \frac{1}{3}\left(\min \left\{x_{2}, z\right\}-y\right)=F\left(x_{2}, y, z\right) .
$$

Therefore, $F(x, y, z)$ is monotone $g$-non-decreasing in $x$. Similarly, we may show that $F(x, y, z)$ is monotone $g$-non-decreasing in $z$ and monotone $g$-nonincreasing in $y$. To prove (5), given $x, y, z, u, v, w \in X$ with $g x \geq g u, g y \leq g v$ and $g z \geq g w$. Then $\frac{3}{4} x \geq \frac{3}{4} u, \frac{3}{4} y \leq \frac{3}{4} v$ and $\frac{3}{4} z \geq \frac{3}{4} w$. Hence $x \geq u, y \leq v$ and $z \geq w$. So, we have the following cases:

Case 1: $y \geq \min \{x, z\}$ and $v \geq \min \{u, w\}$. Here, we have

$$
\begin{aligned}
& d(F(x, y, z), F(u, v, w))=0 \\
\leq & \frac{1}{2} \max \{d(g x, g u), d(g y, g v), d(g z, g w), d(F(x, y, z), g x), \\
& d(F(z, y, x), g x), d(F(u, v, w), g u), d(F(w, v, u), g w)\} .
\end{aligned}
$$

Case 2: $y \geq \min \{x, z\}$ and $v<\min \{u, w\}$. This case is impossible since

$$
y \leq v<\min \{u, w\} \leq \min \{x, z\} .
$$

Case 3: $y<\min \{x, z\}$ and $v \geq \min \{u, w\}$.

Suppose $w \leq v$, then $w-y \leq v-y$ and hence

$$
\begin{aligned}
& \min \{x, z\}-y \\
\leq & z-y=z-w+w-y \\
\leq & z-w+v-y \\
= & \frac{4}{3}\left[\frac{3}{4}(z-w)+\frac{3}{4}(v-y)\right] \\
= & \frac{4}{3}[(g z-g w)+(g v-g y)] \\
= & \frac{4}{3}[d(g z, g w)+d(g v, g y)] \\
\leq & \frac{8}{3} \max \{d(g y, g v), d(g z, g w)\} \\
\leq & \frac{8}{3} \max \{d(g x, g u), d(g y, g v), d(g z, g w), d(F(x, y, z), g x) \\
& d(F(z, y, x), g x), d(F(u, v, w), g u), d(F(w, v, u), g w)\} .
\end{aligned}
$$


Therefore,

$$
\begin{aligned}
& d(F(x, y, z), F(u, v, w)) \\
= & d\left(\frac{1}{3}(\min \{x, z\}-y), 0\right) \\
= & \frac{1}{3}(\min \{x, z\}-y) \\
\leq & \frac{8}{9} \max \{d(g x, g u), d(g y, g v), d(g z, g w), d(F(x, y, z), g x), \\
& d(F(z, y, x), g x), d(F(u, v, w), g u), d(F(w, v, u), g w)\} .
\end{aligned}
$$

Suppose $u \leq v$, then $u-y \leq v-y$ and hence

$$
\begin{aligned}
& \min \{x, z\}-y \\
\leq & x-y \\
= & x-u+u-y \\
\leq & x-u+v-y \\
= & \frac{4}{3}\left[\frac{3}{4}(x-u)+\frac{3}{4}(u-y)\right] \\
= & \frac{4}{3}[(g x-g u)+(g u-g y)] \\
= & \frac{4}{3}[d(g x, g u)+d(g v, g y)] \\
\leq & \left.\frac{8}{3} \max \{d(g x, g u), d(g y, g v))\right\} \\
\leq & \frac{8}{3} \max \{d(g x, g u), d(g y, g v), d(g z, g w), d(F(x, y, z), g x) \\
& d(F(z, y, x), g x), d(F(u, v, w), g u), d(F(w, v, u), g w)\} .
\end{aligned}
$$

Therefore,

$$
\begin{aligned}
& d(F(x, y, z), F(u, v, w)) \\
= & d\left(\frac{1}{3}(\min \{x, z\}-y), 0\right) \\
= & \frac{1}{3}(\min \{x, z\}-y) \\
\leq & \frac{8}{9} \max \{d(g x, g u), d(g y, g v), d(g z, g w), d(F(x, y, z), g x), \\
& d(F(z, y, x), g x), d(F(u, v, w), g u), d(F(w, v, u), g w)\} .
\end{aligned}
$$

Case 4: $y<\min \{x, z\}$ and $v<\min \{u, w\}$. 
Since $x \geq u$ and $z \geq w$, then $\min \{x, z\} \geq \min \{u, w\}$. Thus

$$
\begin{aligned}
& d(F(x, y, z), F(u, v, w)) \\
= & d\left(\frac{1}{3}(\min \{x, z\}-y), \frac{1}{3}(\min \{u, w\}-v)\right) \\
= & \frac{1}{3}|(\min \{x, z\}-\min \{u, w\})+(v-y)| \\
= & \frac{1}{3}[(\min \{x, z\}-\min \{u, w\})+(v-y)]
\end{aligned}
$$

If $\min \{u, w\}=u$, then $\min \{x, z\}-\min \{u, w\} \leq x-u$, and hence

$$
\begin{aligned}
& d(F(x, y, z), F(u, v, w)) \\
\leq & \frac{1}{3}[(x-u)+(v-y)] \\
= & \left(\frac{1}{3}\right)\left(\frac{4}{3}\right)\left[\frac{3}{4}(x-u)+\frac{3}{4}(v-y)\right] \\
= & \frac{4}{9}[(g x-g u)+(g v-g y)] \\
= & \frac{4}{9}[d(g x, g u)+d(g y, g v)] \\
\leq & \frac{8}{9} \max \{d(g x, g u), d(g y, g v)\} \\
\leq & \frac{8}{9} \max \{d(g x, g u), d(g y, g v), d(g z, g w), d(F(x, y, z), g x) \\
& d(F(z, y, x), g x), d(F(u, v, w), g u), d(F(w, v, u), g w)\} .
\end{aligned}
$$

If $\min \{u, w\}=w$, then $\min \{x, z\}-\min \{u, w\} \leq z-w$, and hence

$$
\begin{aligned}
& d(F(x, y, z), F(u, v, w)) \\
\leq & \frac{1}{3}[(z-w)+(v-y)] \\
= & \left(\frac{1}{3}\right)\left(\frac{4}{3}\right)\left[\frac{3}{4}(z-w)+\frac{3}{4}(v-y)\right] \\
= & \frac{4}{9}[(g z-g w)+(g v-g y)] \\
= & \frac{4}{9}[d(g z, g w)+d(g y, g v)] \\
\leq & \frac{8}{9} \max \{d(g y, g v), d(g z, g w)\} \\
\leq & \frac{8}{9} \max \{d(g x, g u), d(g y, g v), d(g z, g w), d(F(x, y, z), g x), \\
& d(F(z, y, x), g x), d(F(u, v, w), g u), d(F(w, v, u), g w)\} .
\end{aligned}
$$


To prove (6), suppose there exist $j, k, l \in[0,1)$ with $j+k+l<1$ such that

$$
d(F(x, y, z), F(u, v, w)) \leq j d(x, u)+k d(y, v)+l d(z, w)
$$

holds for all $x \geq u, y \leq v$ and $z \geq w$. Then

$$
\begin{aligned}
& d(F(1,0,1), F(0,0,1))=d\left(\frac{1}{3}, 0\right)=\frac{1}{3} \leq j, \\
& d(F(1,0,1), F(1,1,1))=d\left(\frac{1}{3}, 0\right)=\frac{1}{3} \leq k,
\end{aligned}
$$

and

$$
d(F(1,0,1), F(1,0,0))=d\left(\frac{1}{3}, 0\right)=\frac{1}{3} \leq l .
$$

From (24), (25), and (26), we have $j+k+l \geq 1$, a contradiction.

To prove (7), suppose there exist $j, k, l \in[0,1)$ with $j+k+l<1$ such that

$$
d(F(x, y, z), F(u, v, w)) \leq j d(g x, g u)+k d(g y, g v)+l d(g z, g w)
$$

holds for all $g x \geq g u, g y \leq g v$ and $g z \geq g w$. Then

$$
\begin{aligned}
& d(F(1,0,1), F(0,0,1))=d\left(\frac{1}{3}, 0\right)=\frac{1}{3} \leq \frac{3}{4} j, \\
& d(F(1,0,1), F(1,1,1))=d\left(\frac{1}{3}, 0\right)=\frac{1}{3} \leq \frac{3}{4} k,
\end{aligned}
$$

and

$$
d(F(1,0,1), F(1,0,0))=d\left(\frac{1}{3}, 0\right)=\frac{1}{3} \leq \frac{3}{4} l .
$$

From (27), (28), and (29), we have $j+k+l \geq \frac{12}{9}$, a contradiction.

Thus by Theorems 2.1 and 2.3, $F$ and $g$ have a tripled coincidence point. Here, $(0,0,0)$ is the tripled coincidence point of $F$ and $g$.

\section{Remarks:}

1. Example 2.1 does not satisfy condition 1 of Theorem 1.1 (Theorem 7 of $[3])$.

2. Example 2.1 does not satisfy condition 2 of Theorem 1.3 (Theorem 4 of $[4])$.

3. Theorem 1.3 (Theorem 4 of [4]) is a special case of Corollaries 2.2 and 2.7 .

4. Theorem 1.1 (Theorem 7 of [3]) is a special case of Corollary 2.5.

5. Theorem 1.2 (Theorem 8 of [3]) is a special case of Corollary 2.10. 


\section{References}

[1] T. G. Bhaskar, V. Lakshmikantham, Fixed point theorems in partially ordered metric spaces and applications, Nonlinear Anal. 65 (2006), 13791393.

[2] V. Lakshmikantham, Lj. Ćirić, Coupled fixed point theorems for nonlinear contractions in partially ordered metric spaces, Nonlinear Anal. 70 (2009), $4341-4349$.

[3] V. Berinde, M. Borcut, Tripled fixed point theorems for contractive type mappings in partially ordered metric spaces, Nonlinear Anal. 74 (2011), 4889-4897.

[4] M. Borcut, V. Berinde, Tripled coincidence theorems for contractive type mappings in partially ordered metric spaces, Appl. Math. Comput. 218 (2012), 5929-5936.

[5] H. Aydi, M. Postolache, W. Shatanawi, Coupled fixed point results for (psi,phi)-weakly contractive mappings in ordered G-metric spaces, Comput. Math. Appl. 63 (2012), 298-309.

[6] H. Aydi, E. Karapinar, M. Postolache, Tripled coincidence point theorems for weak phi-contractions in partially ordered metric spaces, Fixed Point Theory Appl., Vol. 2012, ID: 2012:44, 12 pp.

[7] V. Berinde, Coupled coincidence point theorems for mixed monotone nonlinear operators, Comput. Math. Appl. 64 (2012), 1770-1777.

[8] V. Berinde, Coupled fixed point theorems for $\phi$-contractive mixed monotone mappings in partially ordered metric spaces, Nonlinear Anal. 75 (2012), 3218-3228.

[9] S. Chandok, Z. Mustafa, M. Postolache, Coupled common fixed point theorems for mixed g-monotone mappings in partially ordered G-metric spaces, U. Politeh. Buch. Ser. A, 75 (2013), No. 4, 13-26.

[10] D. Dorić, Z. Kadelburg, S. Radenović, Coupled fixed point results for mappings without mixed monotone property, Appl. Math. Lett. 25 (2012), 1803-1808.

[11] B. S. Choudhury, N. Metiya, M. Postolache, A generalized weak contraction principle with applications to coupled coincidence point problems, Fixed Point Theory Appl., Vol. 2013, ID: 2013:152, 21 pp. 
[12] N. V. Luong, N. X. Thuan, Coupled fixed points in partially ordered metric spaces and applications, Nonlinear Anal. 72 (2011), 983-992.

[13] Z. Golubović, Z. Kadelburg, S. Radenović, Coupled coincidence points of mappings in ordered partial metric spaces, Abstr. Appl. Anal. 2012 (2012), art. no. 192581.

[14] H. K. Nashine and W. Shatanawi, Coupled common fixed point theorems for a pair of commuting mappings in partially ordered complete metric spaces, Comput. Math. Appl. 62 (2011), 1984-1993.

[15] H. K. Nashine, Z. Kadelburg, S. Radenović, Coupled common fixed point theorems for w-compatible mappings in ordered cone metric spaces, Appl. Math. Comput. 218 (2012), 5422-5432.

[16] B. Samet, Coupled fixed point theorems for a generalized Meir-Keeler contraction in partially ordered metric spaces, Nonlinear Anal. 72 (2010), 4508-4517.

[17] B. Samet, C. Vetro, Coupled fixed point, $F$-invariant set and fixed point of $N$-order, Ann. Funct. Anal. 1 (2) (2010), 46-56.

[18] B. Samet, C. Vetro, Coupled fixed point theorems for multi-valued nonlinear contraction mappings in partially ordered metric spaces, Nonlinear Anal. 74 (2011), 4260-4268

[19] B. Samet, H. Yazidi, Coupled fixed point theorems in partially ordered $\varepsilon$-chainable metric spaces, TJMCS. 1 (3) (2010), 142-151.

[20] W. Shatanawi, A. Pitea, Fixed and coupled fixed point theorems of omega-distance for nonlinear contraction. Fixed Point Theory Appl., Vol. 2013, ID: 2013:275, 16 pp.

[21] W. Shatanawi, A. Pitea, Omega-distance and coupled fixed point in Gmetric spaces. Fixed Point Theory Appl., Vol. 2013, Article ID: 2013:208, 15 pp.

[22] W. Shatanawi, A. Pitea, Some coupled fixed point theorems in quasipartial metric spaces. Fixed Point Theory Appl., Vol. 2013, ID: 2013:153, 13 pp.

[23] W. Shatanawi, Z. Mustafa, On coupled random fixed point results in partially ordered metric spaces, Matematicki Vesnik, 64 (2012), 139-146.

[24] W. Shatanawi, Coupled fixed point theorems in generalized metric spaces, Hacettepe J. Mathematics and Statistics, Volume 40 (3) (2011), 441-447. 
[25] W. Shatanawi, Fixed point theorems for nonlinear weakly C-contractive mappings in metric spaces, Math. Comput. Modelling 54 (2011), 28162826, doi:10.1016/j.mcm.2011.06.069

[26] J. Matkowski, Fixed point theorems for mappings with a contractive iterate at a point, Proceedings of the American Mathematical Society, 62 (2) (1977), pp. 344-348.

Wasfi SHATANAWI,

Department of Mathematics,

Hashemite University, Zarqa-Jordan,

Email: swasfi@hu.edu.jo

Mihai POSTOLACHE,

Department of Mathematics and Informatics,

University Politehnica of Bucharest,

313 Splaiul Independenȩti, Romania.

Email: mihai@mathem.pub.ro

Zead MUSTAFA,

Department of Mathematics,

Hashemite University, Zarqa-Jordan,

Email: zmagablh@hu.edu.jo 
